\title{
Role of Math in the Working of the Internet
}

\author{
Arshad Ahamad \\ University Institute of Sciences \\ Department of Mathematics, Chandigarh University \\ Ghuruan, Mohali, Punjab 140413, India \\ Arshadsjd@gmail.com
}

\begin{abstract}
The goal of many mathematicians since the dawn of time has been to apply mathematics to practical applications and to also derive the mathematics behind many everyday things. Although we seldom have such pursuits, many everyday things have been affected by the principles of math. And things like the internet, run on fundamental mathematics principles, and hardly any credit is given to the subject. This paper aims to uncover contributions of everyday Math in the working of the internet. From encryption and decryption all the way to how search engines to index various web pages online, if one looks hard enough, concepts related to mathematics are bound to pop up. This paper also sheds light on various concepts taught in higher education that are often forgotten and only treated as something solely scholastic, but in reality, has a lot of applications in real life.
\end{abstract}

Keywords-Prime Numbers, Encryption, Decryption, Algorithm, Eigen vectors, SEO

\section{INTRODUCTION}

Mathematics: a word that doesn't always spark the common man's curiosity. As Walter Willinger rightly titled his paper about mathematics and the internet as: A source of enormous confusion and great potential [1]. This paper aims to explore the prospects, the applications and various architectures of the Internet that revolves around Mathematics. Unlike the traditional educative approach towards mathematics, where it is just a part of the syllabus and not thought of in terms of real-world applications, this paper discusses multiple areas where even basic concepts of mathematics are the backbone of various areas in the working of the Internet. It is quite obvious that the internet has become a playground for testing various theorems and algorithms for many programmers and mathematicians. Even as an end-user of a simple Application, when one thinks of the workings behind it, there is without a doubt mathematics involved in the structure of things. There are many such areas where mathematics is crucial, but it is a common belief that learning mathematics is something for the sake of school curriculum. This ends up diminishing the intrigue for mathematics itself. From great mathematicians like Pythagoras, whose theorems are taught to this very day to Al-Kawarizmi who invented the algorithm, the foundation of any computer program, mathematics and its applications are always tightly bound in our lives.

\section{A. Understanding its involvement in our daily lives}

Mathematics has been of extraordinary importance since old occasions. The realities of the world were engraved in Ancient Greece, where Plato's thoughts and Pythagoras' hypotheses are still scholarly and discussed today. Not exclusively is math theoretically lovely yet additionally essentially valuable. "Math" as we call it, today is a significant educational program and is inescapable with respect to its part in worldwide schooling. [2] There are 3 fundamental degrees of advantages depicted in Mathematics: Emotional levels like the magnificence of fractals and evenness, Cognitive level, for example, basic reasoning and above all the pragmatic level which incorporates the real factors, ideas and cycles.

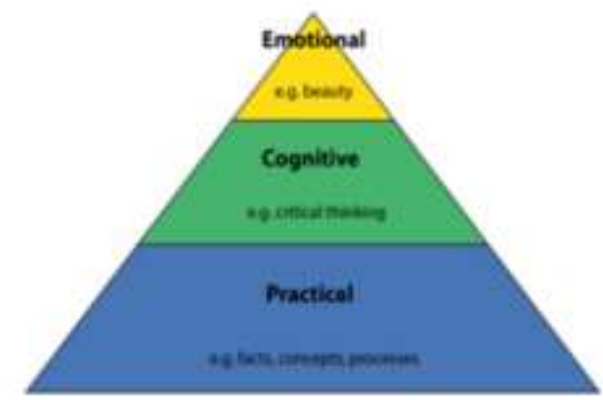

Fig. 1. The 3 levels of benefit which is used as reference for the mathematics curriculum. Source: Charles Fade

Without a doubt, the main degree of numerical advantages lies in the reasonable level. They depict the basic facts of our reality. [3] The potential situations that can be envisioned are unending. A large portion of physical science and designing, climate determining, material science, signal preparing, Internet, security, financial exchange calculations, recreations, Aircraft and space transports, Microbiology, Seismic wave engendering, atomic physical science, discourse acknowledgment, radars and satellites, use mathematics and the rundown goes on. Among these are numerous principal ideas instructed in secondary schools and other higher training, there are numerous people who don't understand where it is being applied.

\section{B. Its Practical Applications throughout histroy}

As referenced previously, the job of math can be followed back to old Greece as Plato conjectured in the seventh book of the republic, Plato portrays learning functional arithmetic as coming to pass its applications to simply shippers and retailers, however for the actual spirit. He didn't believe the learning of science to be saved solely for the privileged of the Populus. This old view remains constant even today as understudies need to keep on learning math alongside its application. It is an essential for building up certain problem solving skills and a sense of Quantitative reasoning. Arithmetic as a subject began in post-progressive France in the 
late eighteenth century where, dependent on conviction, the man was decided as sane in the event that he learned Mathematics. Indeed, even while this origination started to vanish, the actual subject stayed a significant piece of the French educational plan as a component of its advanced education, as a piece of Status, and a vehicle for social separation. [2]

The current spot of arithmetic is the educational programs all throughout the planet accepting a cumulation of different thoughts in history for the instructing of Mathematics. The exhibition of understudies in numerical tests is utilized to make claims about the situation of different states and countries. Schooling frameworks and individual test scores profoundly incorporate arithmetic as a significant subject which makes it a basically significant factor in deciding school acknowledgment. [2]

This, nonetheless, ought not be the cutoff and degree of the motivation behind learning Mathematics. Not only does this make Mathematics something you leave behind in your schooling years, it puts the subject on a bad light making the students who studied it without ever thinking of its practical application to always frown upon when hearing the word.

The internet is a great area to look around and discover the underlying concepts of this subject. Some of the research and discussions over this topic and various areas and papers where mathematics was used as the main area of approach towards solving a problem. The web is from one side of the planet to the other, assisting billions with associating together. It can't be disregarded that there are various ideas on the web that are advancements of numerous ideas of arithmetic.

\section{MATHEMATICAL CONCEPST USED TODAY}

\section{A. Use of Prime Numbers in Internet Security}

Any site you visit will have a specific lock image in the location bar of your browser if the site is secure. This implies that the site utilizes an encryption framework, which implies that all data that is passed to from the web server where the information dwells is encoded such that none but the server can decode what the actual information is.

\section{Q https://eu.bbc...}

\section{Fig. 2. The lock symbol next to websites signifies encryption}

To understand how this encryption works, we again look back into grade school amthematics. An indivisible number is any number that has no further factors other than the actual number. For instance, 5, 7, 13, 17, and so on are indivisible numbers. Different numbers like $4(2 * 2), 6(3 * 2)$ are NonPrime or composite numbers since they have different components. To a degree, you can perceive a number is prime. It is not difficult to say that 23 and 113 are indivisible numbers. But a computer can look at much larger numbers and determine if a number is prime or not. In any case, encryption on the web where individuals store bank passwords and store private data can't utilize such little numbers.

Consider the number given underneath.

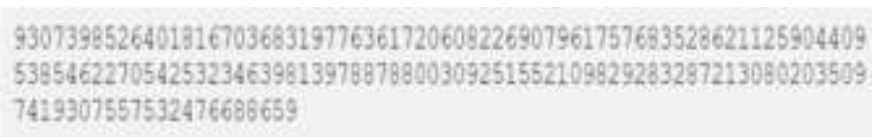

For a person, the number may take a ton of time to unravel if prime or not to mention rattle off the entirety of its elements. Notwithstanding, for a PC, it is a simple errand. Despite the fact that more slow frameworks may take some time, as long as the private key and public key is known, the number can be interpreted. This is discussed further below.

There are numerous sorts of encryption which incorporate symmetric encryption, uneven encryption, Trapdoors, and so forth The most widely recognized sort of encryption is RSA encryption. [3] Although it is the greater part extremely old, it is still broadly trusted and utilized due to one explanation: Prime Numbers. [3]

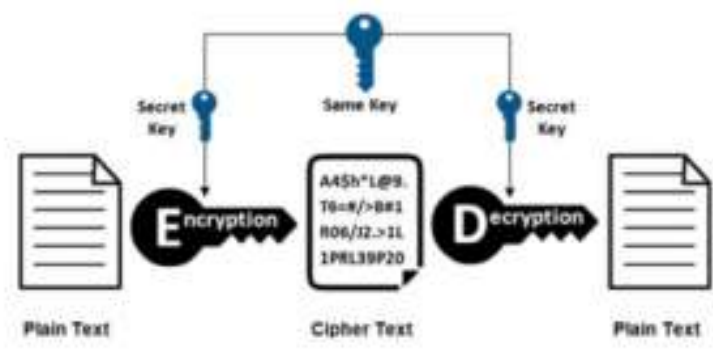

Fig. 3. Symmetric Encryption. Image Credit: Ons Jallouli via ResearchGate[6]

The fundamental idea of any encryption calculation is that the client sends plain content which is justifiable to him (ie. English). The content is changed over to a result of 2 primes guess $\mathrm{x}$ and $\mathrm{y}$. The upsides of $\mathrm{x}$ and $\mathrm{y}$ are known exclusively by the worker PC which is facilitating the site. The client just knows the result of the numbers p. Subsequently, at whatever point the message is prepared to communicate, it is changed over utilizing the result of 2 primes as the encryption key. So any individual who blocks these messages can't comprehend it. To comprehend the message, it again should be decoded utilizing the mysterious key that is a blend of $\mathrm{x}$ and $\mathrm{y}$.

These keys are known as public key and private key. The public key being the normal product of 2 primes which is known by the users on the internet. The private key is the product 2 very large prime numbers that is known only to the servers. For example, Whatsapp utilizes 256-cycle encoders to send and get messages. This implies that the public key can be a number that is 256 digits huge, practically too cruel to tackle. 


\section{B. Use of EigenVectors in Google's search Algorithms}

No one had thought Google would be an organization as large as it is today when Larry Page initially started the organization with 41 workers. And at that time, till now a major challenge for such service providers have been providing the best results to any query submitted on a search engine and also hyperlinking each page with information to other pages on the internet.[7] It was when the organization began building its reputation that Larry Page distributed his paper on the Google page positioning calculation [4]. He named the calculation PageRank. It was a technique for rating pages both equitably and precisely, to gauge the human interest it made and its significance to them. Present day web indexes channel query items considering a certain something: to give the best outcomes. Straightforward page positioning calculations would not get the job done for an overall web client.

Probably the best calculation made was the PageRank algorithm. For instance, on the off chance that we have a page called home and a connection to a site page called Page1, this would make Page1 pertinent to our theme. Assuming there is a great deal of pages that connect to Page1, it would suggest that Page1 is significant. Also, if Page1 had only one backlink, yet it came from a vital site like Google, MSN, and so forth then the significance is moved, this means, Page 1 is significant once more. Considering this, we picture the web as a coordinated chart. [4].

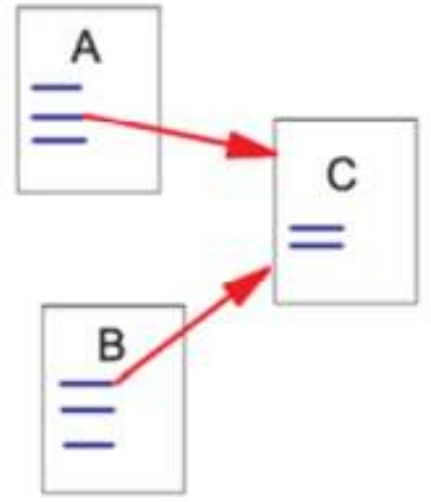

The page ranking algorithm is much more complex system that positions each page into a matrix. Matrices and determinants are another concept that are prevalent in the Internet's working. Not only did the pages get stored in a matrix, but to determine the best search results out of the matrix of web-pages, Eigenvectors were calculated. This gave rise to one of the most fundamental and mathematical algorithms of Google for ranking webpages on the Internet.

So, could it be that happens to the network of pertinent pages that assisted Google with turning into the most mainstream web search tool ever? The outcome is otherwise called the $\$ 250000000000$ Eigenvector. Although a lot of modifications have been done and the modern search uses many other algorithms, the fundamental concept of Google's success doesn't change. The eigenvectors are determined to comprehend the likelihood of the client winding up on a specific site. It shows the probabilities of all the website page significance. [4]

In light of which page has the most elevated likelihood, it is positioned in the google search. The calculation has gone through loads of enhancements over the years. Joined with AI, it has made Google Search what is today

\section{MATHEMATICS IN THE FABRIC OF THE INTERNET}

The Internet is an industry that is multiplying in size each year. To a certain extent, one could call the internet a massive organism whose true capabilities are far from being realized. It has without a doubt experienced intriguing advancements previously. As Anthony Michael Rutkowski puts it, " the web is its own transformation." [5] The insurgency of the web is without a doubt influencing ideas of Mathematics. Only, when we realize its uses, it gives us an appreciation for the basic use of the lovely subject. In quest for building up the organization and in understanding what kind of math is done, Internet Engineering turns into a Massive field for exciting and new research, opening new doors in the field of Mathematical Sciences.

In its beginning phase, the Internet stressed designing and experimentation. It was moderately less worried about Mathematics and hypothesis. Truth be told, some members online would go on to say that the Internet is the thing that it is today since it " overlooked math". [5] This anyway couldn't possibly be more off-base as it has been expressed before: the reason behind any safe webpage, and the calculate ion behind Google, which is visited 3.9 Million times each moment all have significant numerical ideas driving their working.

Till this date, the main role that math played in network science has only been to put physicists' largely empirical research findings into more solid grounds by means of meticulous proofs. [1,8]. Although this presents the forementioned role as something highly advanced, the truth is that as we look starting from the surface, the basic concepts of mathematics that we learnt at a young age come to light. In this review, a retrospective view was presented on the mathematical treatment of the working behind the Internet. Our motive was to establish that Mathematics is still very relevant and the internet which surrounds us and has revolutionized the way we live our lives, was a clearly good case study.

\section{CONCLUSION}

We have shown that numerical ideas are applied to the basic and complex pieces of our regular daily existences. This investigation was directed to reveal insight into a portion of the vital applications even the fundamental ideas of mathematics have in reality. Without a doubt, the knowledge pf numerical abilities are vital as it gives another viewpoint to the large numbers of moving parts in a normal individual's life. Numerical ideas, for example, indivisible numbers are utilized in giving web security and different ideas like Eigenvectors are utilized as an establishment in making a billion-dollars' worth pursuit calculation. It is imperative to ensure that the Mathematics taught today readies the understudies with the information on how what they realized is helpful on the planet. The functional advantages of math are doubtlessly, too huge to overlook.

\section{ACKNOWLEDGMENT}

I thank Dr. Richa Sharma, (Department of Mathematics, Chandigarh University) for her guidance and useful discussion. 


\section{REFERENCES}

[1] Willinger, Walter, David Alderson, and John C. Doyle. "Mathematics and the internet: A source of enormous confusion and great potential." Notices of the American Mathematical Society 56.5 (2009): 586-599.

[2] Maya Bailik, Alexandre Kabbach, Mathematics for the 21st century, Bill and Melinda Gates Foundation, July 2014

[3] Wijaya, Ariyadi. (2020). The role of mathematics teacher in the digital era. Journal of Physics: Conference Series. 1581. 012069. 10.1088/1742$6596 / 1581 / 1 / 012069$

[4] Page, Lawrence and Brin, Sergey and Motwani, Rajeev and Winograd, Terry (1999) The PageRank Citation Ranking: Bringing Order to the Web. Technical Report. Stanford InfoLab. SIDL-WP-1999-0120.

[5] Willinger, V Paxson, Where Mathematics meets the Internet, Notices of the AMS, 1998, ams.org

[6] JALLOULI, Ons. (2017). Chaos-based security under real-time and energy constraints for the Internet of Things.

[7] Sharma, Dilip Kumar, and A. K. Sharma. "A comparative analysis of web page ranking algorithms." International Journal on Computer Science and Engineering 2.08 (2010): 2670-2676

[8] B. Bollobás and O. Riordan, Mathematical results on scale-free random graphs, Handbook of Graphs and Networks (S. Bornholdt and H. G. Schuster, eds.), Wiley-VCH, Weinheim, 2002. 